\title{
ISOMETRIC DEFORMATIONS OF MINIMAL SURFACES IN $\mathbb{S}^{4}$
}

\author{
THEODOROS VLACHOS
}

\begin{abstract}
We provide an elementary proof of the fact that the space of all isometric minimal immersions $f: M \rightarrow \mathbb{S}^{4}$ of a 2dimensional Riemannian manifold $M$ into $\mathbb{S}^{4}$ with the same normal curvature is, up to congruence, either finite or a circle. Furthermore, we show that if $M$ is compact and the Euler number of the normal bundle of $f$ is nonzero, then there exist at most finitely many noncongruent isometric minimal immersions of $M$ into $\mathbb{S}^{4}$ with the same normal curvature.
\end{abstract}

\section{Introduction}

A classical question about isometric immersions is to decide if given an isometric immersion $f: M \rightarrow N$, this is, up to ambient isometries, the unique way of immerse isometrically the Riemannian manifold $M$ into the Riemannian manifold $N$. When $f$ is a minimal immersion, one can ask if this is the unique isometric minimal immersion of $M$ into $N$. If this is the case, $f$ is called minimally rigid. The rigidity aspects of minimal hypersurfaces in space forms have drawn several author's attention. A conclusive result was given Dajczer and Gromoll [5] for complete minimal hypersurfaces.

It is interesting to determine whether a given minimal surface can be deformed in a nontrivial way. Choi, Meeks and White [4] proved a rigidity result for complete minimal surfaces in $\mathbb{R}^{3}$. The case where the Euclidean space is replaced by a sphere is more difficult. Barbosa [2] proved that minimally immersed 2 -spheres in a sphere are minimally rigid, while according to Ramanathan [12] any compact minimal surface in $\mathbb{S}^{3}$, allows at most finitely many noncongruent isometric minimal surfaces.

We are interested in isometric deformations of oriented minimal surfaces $f: M \rightarrow \mathbb{S}^{4}$ which preserve the normal curvature. If $M$ is simply connected,

Received March 26, 2014; received in final form October 9, 2014.

2010 Mathematics Subject Classification. 53A10, 53C42. 
then there exists a $2 \pi$-periodic one-parameter family $f_{\theta}$ of isometric minimal immersions preserving the normal curvature, the associated family. This family is obtained by rotating the second fundamental form of $f$ and then integrate the system of Gauss, Codazzi and Ricci equations. It is trivial, in the sense that each $f_{\theta}$ is congruent to $f$, if $f$ is superminimal (cf. [14]). Thus the rigidity for simply connected minimal surfaces fails in a natural way, and consequently the rigidity problem for minimal surfaces has a global nature. The above procedure cannot be carried out if the fundamental group is nontrivial. Inspired by a recent paper due to Smyth and Tinaglia [13], we provide elementary proofs of the following results concerning the space of isometric deformations of minimal surfaces in $\mathbb{S}^{4}$.

THEOREM 1. Let $f: M \rightarrow \mathbb{S}^{4}$ be an isometric minimal immersion of a 2-dimensional Riemannian manifold $M$ into $\mathbb{S}^{4}$ with normal curvature $K_{N}$. Then, up to congruence, the space of all isometric minimal immersions of $M$ into $\mathbb{S}^{4}$ with normal curvature $K_{N}$ is either finite or a circle.

THEOREM 2. Let $f: M \rightarrow \mathbb{S}^{4}$ be an isometric minimal immersion of a compact oriented 2-dimensional Riemannian manifold $M$ into $\mathbb{S}^{4}$ with normal curvature $K_{N}$. If the Euler number of the normal bundle of $f$ is nonzero, then there exist at most finitely many noncongruent isometric minimal immersions of $M$ into $\mathbb{S}^{4}$ with normal curvature $K_{N}$.

The Euler number of the normal bundle of $f$ is nonzero if and only if its self-intersection number $q$ is nonzero ([11, Cor. 3.2]). It is known (cf. [9]) that for any compact nonsuperminimal surface of genus $g$ we have $|q| \leq 2(g-1)$. Thus $g \geq 2$ if the Euler number of its normal bundle is nonzero. All compact superminimal surfaces in $\mathbb{S}^{4}$, which are not totally geodesic, have normal bundle with nonzero Euler number. For all these surfaces, the number $N$ of noncongruent isometric minimal immersions into $\mathbb{S}^{4}$ is $N=1$. We do not know any examples with $N \geq 2$.

An immediate application of Theorem 2 is the following.

Corollary 3. Let $f: M \rightarrow \mathbb{S}^{4}$ be compact minimal surface whose normal bundle has nonzero Euler number. If $M$ admits a one parameter group of isometries $\varphi_{t}$, that preserve the normal curvature, then there exists a one parameter group of isometries $\tau_{t}$ of $\mathbb{S}^{4}$ such that $f \circ \varphi_{t}=\tau_{t} \circ f$ for all $t \in \mathbb{R}$.

\section{Preliminaries}

Let $f: M \rightarrow \mathbb{S}^{4}$ be a minimal surface, that is, an isometric minimal immersion of a connected oriented 2-dimensional Riemannian manifold $M$, with normal bundle $N f$ and second fundamental form $B$. We view $M$ as a Riemann surface with complex structure determined by the metric and the orientation. The complexified tangent bundle $T M \otimes \mathbb{C}$ is decomposed into the eigenspaces of the complex structure $J$, called $T^{\prime} M$ and $T^{\prime \prime} M$, corresponding 
to the eigenvalues $i$ and $-i$. The second fundamental form can be complex linearly extended to $T M \otimes \mathbb{C}$ with values in the complexified bundle $N f \otimes \mathbb{C}$ and then decomposed into its $(p, q)$-components, $p+q=2$, which are tensor products of $p$ many 1-forms vanishing on $T^{\prime \prime} M$ and $q$ many 1-forms vanishing on $T^{\prime} M$. Since $f$ is minimal, for any local complex coordinate $z=u+i v$, we have

$$
B=B^{(2,0)}+B^{(0,2)},
$$

where

$$
B^{(2,0)}=B\left(\frac{\partial}{\partial z}, \frac{\partial}{\partial z}\right) d z^{2}, \quad B^{(0,2)}=\overline{B^{(2,0)}} \quad \text { and } \quad \frac{\partial}{\partial z}=\frac{1}{2}\left(\frac{\partial}{\partial u}-i \frac{\partial}{\partial v}\right) .
$$

The Hopf differential is the differential form of type $(4,0)$

$$
\Phi:=\left\langle B^{(2,0)}, B^{(2,0)}\right\rangle .
$$

For any local orthonormal frame field $\left\{e_{j}\right\}$ along $f$, such that $\left\{e_{1}, e_{2}\right\}$ are tangent with dual coframe $\left\{\omega_{1}, \omega_{2}\right\}$, we put

$$
H_{\alpha}=\left\langle B\left(e_{1}, e_{1}\right), e_{\alpha}\right\rangle+i\left\langle B\left(e_{1}, e_{2}\right), e_{\alpha}\right\rangle, \quad \alpha=3,4 \quad \text { and } \quad \varphi=\omega_{1}+i \omega_{2} .
$$

We easily obtain

$$
\Phi=\frac{1}{4}\left(\bar{H}_{3}^{2}+\bar{H}_{4}^{2}\right) \varphi^{4}
$$

The curvature ellipse of $f$ at $x \in M$, is

$$
\mathcal{E}(x)=\left\{B(X, X): X \in T_{x} M,|X|=1\right\} .
$$

The zeros of $\Phi$ are precisely the points where the curvature ellipse is a circle. A minimal surface is called superminimal if $\Phi \equiv 0$. The Codazzi equation implies that $\Phi$ is holomorphic (cf. [3]). Thus either $f$ is superminimal, or the points where the curvature ellipse is a circle are isolated.

The normal curvature $K_{N}$ (cf. [1]) is given by

$$
d \omega_{34}=-K_{N} \omega_{1} \wedge \omega_{2},
$$

or equivalently,

$$
K_{N}=i\left(H_{3} \bar{H}_{4}-\bar{H}_{3} H_{4}\right) .
$$

We note that $\left|K_{N}\right|=2 \kappa \mu$, where $\kappa \geq \mu \geq 0$ are the length of the semi-axes of the curvature ellipse. The length of the second fundamental form satisfies

$$
\|B\|^{2}=2\left(\left|H_{3}\right|^{2}+\left|H_{4}\right|^{2}\right) .
$$

Using the null frame field $\eta=e_{3}+i e_{4}, \bar{\eta}=e_{3}-i e_{4}$ of the complexified bundle $N f \otimes \mathbb{C}$, we have

$$
\left\langle B^{(2,0)}, B^{(2,0)}\right\rangle=\left\langle B^{(2,0)}, \eta\right\rangle\left\langle B^{(2,0)}, \bar{\eta}\right\rangle .
$$

Therefore, we obtain

$$
\Phi=\frac{1}{4}\left(\bar{H}_{3}^{2}+\bar{H}_{4}^{2}\right) \varphi^{4}=\frac{1}{4} k_{+} k_{-} \varphi^{4},
$$


where $k_{ \pm}:=\bar{H}_{3} \pm i \bar{H}_{4}$. The functions $a_{ \pm}:=\left|k_{ \pm}\right|$determine the geometry of the curvature ellipse. Indeed, since the Gaussian curvature $K$ of $M$ is given by $K=1-\|B\|^{2} / 2$, from (1) and (2) we deduce that $a_{ \pm}=\left(1-K \pm K_{N}\right)^{1 / 2}=$ $\kappa \pm \varepsilon \mu$, where $\varepsilon= \pm 1$, depending on the sign of $K_{N}$.

\section{Isometric deformations of minimal surfaces in $\mathbb{S}^{4}$}

3.1. Associated family of simply connected minimal surfaces. Let $f: M \rightarrow \mathbb{S}^{4}$ be a simply connected minimal surface. For each $\theta \in \mathbb{S}^{1}=\mathbb{R} / 2 \pi \mathbb{Z}$, we consider the orthogonal and parallel tensor field

$$
J_{\theta}=\cos \theta I+\sin \theta J
$$

where $I$ is the identity map and $J$ is the complex structure. The symmetric section $\Gamma_{\theta}$ of the homomorphism bundle $\operatorname{Hom}(T M \times T M, N f)$ given by

$$
\Gamma_{\theta}(X, Y):=B\left(J_{\theta} X, Y\right), \quad X, Y \in T M
$$

satisfies the Gauss, Codazzi and Ricci equations with respect to the normal connection $\nabla^{\perp}$ of $f$ (cf. [14], [5], [6]). Hence there exists an isometric minimal immersion $f_{\theta}: M \rightarrow \mathbb{S}^{4}$ with second fundamental form

$$
B^{f_{\theta}}(X, Y)=T_{\theta} \circ B\left(J_{\theta} X, Y\right),
$$

where $T_{\theta}: N f \rightarrow N f_{\theta}$ is a parallel and orthogonal vector bundle isomorphism. The $2 \pi$-periodic family $f_{\theta}$ is the associated family of $f$. It is trivial, in the sense that each $f_{\theta}$ is congruent to $f$, if $f$ is superminimal (cf. [14]).

It is obvious that $f$ and $f_{\theta}$ have the same normal curvature. Eschenburg and Tribuzy [9, Th. 2] proved that any other minimal immersion of $M$ into $\mathbb{S}^{4}$ with normal curvature $K_{N}$ is congruent to some $f_{\theta}$.

3.2. Deformations of nonsimply connected minimal surfaces. Let $f: M \rightarrow \mathbb{S}^{4}$ be a nonsimply connected minimal surface with normal curvature $K_{N}$. Since superminimal surfaces are rigid among superminimal surfaces (cf. [15]), we may assume hereafter that $f$ is not superminimal. Let $g: M \rightarrow \mathbb{S}^{4}$ be another immersed minimal surface with normal curvature $K_{N}$. We consider the covering map $p: \tilde{M} \rightarrow M, \tilde{M}$ being the universal cover of $M$ equipped with the metric and the orientation that makes $p$ an orientation preserving local isometry. Corresponding objects on $\tilde{M}$ are denoted with tilde. The minimal surfaces $\tilde{f}:=f \circ p$ and $\tilde{g}:=g \circ p$ have normal curvature $\tilde{K}_{N}=K_{N} \circ p$. According to [9], $\tilde{g}$ is congruent to some $\tilde{f}_{\theta}$ in the associated family of $\tilde{f}$. Thus, the space of all isometric minimal immersions of $M$ into $\mathbb{S}^{4}$ with normal curvature $K_{N}$ is the set

$$
\mathcal{S}(f):=\left\{\theta \in[0,2 \pi]: \text { there exists } f_{\theta}: M \rightarrow \mathbb{S}^{4} \text { so that } \tilde{f}_{\theta}=f_{\theta} \circ p\right\} .
$$

Clearly $0 \in \mathcal{S}(f)$ and, for each $\theta \in \mathcal{S}(f)$, the normal curvature of $f_{\theta}$ is $K_{N}$.

Lemma 4. For any $\sigma$ in the group $\mathcal{D}$ of deck transformations of the covering map $p: \tilde{M} \rightarrow M$, the minimal surfaces $\tilde{f}_{\theta}$ and $\tilde{f}_{\theta} \circ \sigma$ are congruent. 
Proof. It is enough to prove the existence of an orthogonal and parallel isomorphism between the normal bundles of $\tilde{f}_{\theta}$ and $\tilde{f}_{\theta} \circ \sigma$ that preserves the second fundamental forms. If $T_{\theta}$ is the isomorphism between the normal bundles of $\tilde{f}$ and $\tilde{f}_{\theta}$, then we define the bundle isomorphism

$$
\Sigma_{\theta}: N \tilde{f}_{\theta} \rightarrow N\left(\tilde{f}_{\theta} \circ \sigma\right)
$$

so that $\left.\Sigma_{\theta}\right|_{\tilde{x}}: N_{\tilde{x}} \tilde{f}_{\theta} \rightarrow N_{\tilde{x}}\left(\tilde{f}_{\theta} \circ \sigma\right)$ is given at any point $\tilde{x} \in \tilde{M}$ by

$$
\left.\Sigma_{\theta}\right|_{\tilde{x}}(\xi):=\left.T_{\theta}\right|_{\sigma(\tilde{x})}\left(\left.T_{\theta}^{-1}\right|_{\tilde{x}}(\xi)\right), \quad \xi \in N_{\tilde{x}} \tilde{f}_{\theta} .
$$

The second fundamental forms of $\tilde{f}_{\theta} \circ \sigma$ and $\tilde{f}_{\theta}$ are related by

$$
\left.B^{\tilde{f}_{\theta} \circ \sigma}\right|_{\tilde{x}}(\tilde{v}, \tilde{w})=\left.T_{\theta} \circ B^{\tilde{f}}\right|_{\sigma(\tilde{x})}\left(\tilde{J}_{\theta} \circ d \sigma_{\tilde{x}}(\tilde{v}), d \sigma_{\tilde{x}}(\tilde{w})\right), \quad \tilde{v}, \tilde{w} \in T_{\tilde{x}} \tilde{M},
$$

where $\tilde{J}_{\theta}=\cos \theta \tilde{I}+\sin \theta \tilde{J}$. Since $\sigma$ is a deck transformation, it follows that

$$
\left.B^{\tilde{f}_{\theta} \circ \sigma}\right|_{\tilde{x}}(\tilde{v}, \tilde{w})=\left.\Sigma_{\theta} \circ B^{\tilde{f_{\theta}}}\right|_{\tilde{x}}(\tilde{v}, \tilde{w}) .
$$

Let $\xi=T_{\theta}(\eta)$, where $\eta$ is a section of $N \tilde{f}$. Since $\Sigma_{\theta}(\xi)=T_{\theta}\left(\eta \circ \sigma^{-1}\right) \circ \sigma$, for any $\tilde{X}$ tangent to $\tilde{M}$, we have

$$
\begin{aligned}
\left(\nabla_{\tilde{X}}^{\perp} \Sigma_{\theta}\right) \xi & =\nabla_{\tilde{X}}^{\frac{1}{(}}\left(T_{\theta}\left(\eta \circ \sigma^{-1}\right) \circ \sigma\right)-T_{\theta}\left(\nabla_{\tilde{X}}^{\perp}\left(\eta \circ \sigma^{-1}\right)\right) \circ \sigma \\
& =\left(\nabla_{d \sigma(\tilde{X})}^{\perp} T_{\theta}\left(\eta \circ \sigma^{-1}\right)\right) \circ \sigma-T_{\theta}\left(\nabla_{\tilde{X}}^{\perp}\left(\eta \circ \sigma^{-1}\right)\right) \circ \sigma \\
& =T_{\theta}\left(\nabla_{d \sigma(\tilde{X})}^{\perp}\left(\eta \circ \sigma^{-1}\right)-\nabla_{\tilde{X}}^{\perp}\left(\eta \circ \sigma^{-1}\right)\right) \circ \sigma,
\end{aligned}
$$

where, by abuse of notation, $\nabla^{\perp}$ stands for the normal connection of both $\tilde{f}_{\theta}$ and $\tilde{f}_{\theta} \circ \sigma$. Let $\delta$ be a local section of $N f$ such that $\eta \circ \sigma^{-1}=\delta \circ p$. Now observe that

$$
\nabla_{d \sigma(\tilde{X})}^{\perp}\left(\eta \circ \sigma^{-1}\right)-\nabla_{\tilde{X}}^{\perp}\left(\eta \circ \sigma^{-1}\right)=\nabla_{d p \circ d \sigma(\tilde{X})}^{\perp} \delta-\nabla_{d p(\tilde{X})}^{\perp} \delta=0 .
$$

Therefore $\Sigma_{\theta}$ is parallel, and this completes the proof.

Proof of Theorem 1. Lemma 4 allows us to define a homomorphism $\Phi_{\theta}: \mathcal{D} \rightarrow \operatorname{Isom}\left(\mathbb{S}^{4}\right)$ for each $\theta \in[0,2 \pi]$, such that

$$
\tilde{f}_{\theta} \circ \sigma=\Phi_{\theta}(\sigma) \circ \tilde{f}_{\theta}, \quad \sigma \in \mathcal{D} .
$$

We observe that $\theta \in \mathcal{S}(f)$ if and only if $\Phi_{\theta}(\mathcal{D})=\{I\}$. Assume that $\mathcal{S}(f)$ is infinite. Then there exists a sequence $\left\{\theta_{m}\right\}$ in $\mathcal{S}(f)$ which converges to some $\theta_{0} \in[0,2 \pi]$. From $\Phi_{\theta_{m}}(\mathcal{D})=\{I\}$ for all $m \in \mathbb{N}$, we immediately obtain $\Phi_{\theta_{0}}(\mathcal{D})=\{I\}$. Let $\sigma \in \mathcal{D}$. By applying the Mean Value Theorem to each entry $\left(\Phi_{\theta}(\sigma)\right)_{j k}$ of the corresponding matrix, we have

$$
\frac{d}{d \theta}\left(\Phi_{\theta}(\sigma)\right)_{j k}\left(\stackrel{\circ}{m}_{m}\right)=0
$$

for some $\stackrel{\circ}{m}_{m}$ which lies between $\theta_{0}$ and $\theta_{m}$. By continuity, we obtain

$$
\frac{d}{d \theta}\left(\Phi_{\theta}(\sigma)\right)_{j k}\left(\theta_{0}\right)=0
$$


Applying repeatedly the Mean Value Theorem, we conclude that

$$
\frac{d^{n}}{d \theta^{n}}\left(\Phi_{\theta}(\sigma)\right)_{j k}\left(\theta_{0}\right)=0
$$

any integer $n \geq 1$. Since $\Phi_{\theta}(\sigma)$ is an analytic curve (cf. [8]) in $\operatorname{Isom}\left(\mathbb{S}^{4}\right)$, we infer that $\Phi_{\theta}(\sigma)=I$ for each $\sigma \in \mathcal{D}$, and so $\mathcal{S}(f)=[0,2 \pi]$.

\section{Isometric deformations of compact minimal surfaces}

For the proof of Theorem 2, we need some auxiliary lemmas.

LEMMA 5. Let $f: M \rightarrow \mathbb{S}^{4}$ be a minimal surface which is not contained in any totally geodesic $\mathbb{S}^{3}$. For any $\theta \in \mathcal{S}(f)$ there exists a parallel and orthogonal bundle isomorphism $T_{\theta}: N f \rightarrow N f_{\theta}$ such that the second fundamental forms of $f$ and $f_{\theta}$ are related by

$$
B^{f_{\theta}}(X, Y)=T_{\theta} \circ B^{f}\left(J_{\theta} X, Y\right), \quad X, Y \in T M .
$$

Proof. Since $f$ and $f_{\theta}$ have the same normal curvature, for any simply connected subset $U$ of $M$ there exists a parallel and orthogonal bundle isomorphism $T_{\theta}^{U}:\left.\left.N f\right|_{U} \rightarrow N f_{\theta}\right|_{U}$ such that the second fundamental forms of $\left.f\right|_{U}$ and $\left.f_{\theta}\right|_{U}$ are related by

$$
B^{\left.f_{\theta}\right|_{U}}(X, Y)=T_{\theta}^{U} \circ B^{\left.f\right|_{U}}\left(J_{\theta} X, Y\right), \quad X, Y \in T M .
$$

Let $U, V$ be simply connected subsets of $M$ with $U \cap V \neq \varnothing$. Then we have $T_{\theta}^{U}=T_{\theta}^{V}$ on $U \cap V \backslash M_{0}$, where $M_{0}$ is the set of points where the normal curvature vanishes. Since $M \backslash M_{0}$ is dense in $M$, by continuity, we see that $T_{\theta}^{U}=T_{\theta}^{V}$ on $U \cap V$. Thus $T_{\theta}^{U}$ is globally well-defined.

Lemma $6([15])$. Assume that $f: M \rightarrow \mathbb{S}^{4}$ is not superminimal and let $M_{1}$ be the zero set of $\Phi$. Around each point in $M \backslash M_{1}$, there exist a local complex coordinate $(U, z), U \subset M \backslash M_{1}$ and orthonormal frames $\left\{e_{1}, e_{2}\right\}$ in $T M \mid U$, $\left\{e_{3}, e_{4}\right\}$ in $N f \mid U$ which agree with the given orientations such that

(i) the Riemannian metric of $M$ is given by

$$
d s^{2}=\frac{|d z|^{2}}{\left(\kappa_{1}^{2}-\mu_{1}^{2}\right)^{1 / 2}} \quad \text { and } \quad \frac{\partial}{\partial z}=\frac{e_{1}-i e_{2}}{2\left(\kappa_{1}^{2}-\mu_{1}^{2}\right)^{1 / 4}},
$$

(ii) $e_{3}$ and $e_{4}$ give respectively the directions of the major and the minor axes of the curvature ellipse, and

(iii) $H_{3}=\kappa_{1}, H_{4}=i \mu_{1}$, where $\kappa_{1}$ and $\mu_{1}$ are smooth real functions with $\kappa=\left|\kappa_{1}\right|, \mu=\left|\mu_{1}\right|$. Moreover, the connection and the normal connection forms, with respect to this frame, are given respectively, by

$$
\omega_{12}=-\frac{1}{4} * d \log \left(\kappa_{1}^{2}-\mu_{1}^{2}\right), \quad \omega_{34}=* \frac{\kappa_{1} d \mu_{1}-\mu_{1} d \kappa_{1}}{\kappa_{1}^{2}-\mu_{1}^{2}},
$$

where * stands for the Hodge operator. 
Let $f: M \rightarrow \mathbb{S}^{4}$ be a minimal surface which is not contained in any totally geodesic $\mathbb{S}^{3}$. Assume hereafter that $f$ is not superminimal. For each point $x \in$ $M \backslash M_{1}$, we consider an orthonormal frame $\left\{e_{1}, e_{2}, e_{3}, e_{4}\right\}$ on a neighborhood $U_{x} \subset M \backslash M_{1}$ of $x$ as in Lemma 6 . We note that $\omega_{34}$ cannot vanish on any open subset of $M \backslash M_{1}$. For any $\theta \in \mathcal{S}(f),\left\{e_{1}, e_{2}, T_{\theta} e_{3}, T_{\theta} e_{4}\right\}$ is a frame along $f_{\theta}$, where $T_{\theta}: N f \rightarrow N f_{\theta}$ is the bundle isomorphism of Lemma 5 . We observe that $H_{3}, H_{4}$ and the corresponding functions $H_{3}^{\theta}, H_{4}^{\theta}$ for $f_{\theta}$, associated to the frame $\left\{e_{1}, e_{2}, T_{\theta} e_{3}, T_{\theta} e_{4}\right\}$, satisfy

$$
H_{3}^{\theta}=\exp (-i \theta) H_{3} \quad \text { and } \quad H_{4}^{\theta}=\exp (-i \theta) H_{4} .
$$

Using (4) and the Weingarten formula for $f_{\theta}$, we obtain

$$
\begin{gathered}
\tilde{\nabla}_{E}\left(T_{\theta} e_{3}\right)=-\kappa_{1} \exp (i \theta) d f_{\theta}(\bar{E})+\omega_{34}(E) T_{\theta} e_{4}, \\
\tilde{\nabla}_{E}\left(T_{\theta} e_{4}\right)=i \mu_{1} \exp (i \theta) d f_{\theta}(\bar{E})-\omega_{34}(E) T_{\theta} e_{3},
\end{gathered}
$$

where $E=e_{1}-i e_{2}$ and $\tilde{\nabla}$ stands for the usual connection in the induced bundle $\left(i_{1} \circ f\right)^{*}\left(T \mathbb{R}^{5}\right), i_{1}: \mathbb{S}^{4} \rightarrow \mathbb{R}^{5}$ being the inclusion map.

LEMMA 7. Assume that for $\theta_{j} \in \mathcal{S}(f), j=1, \ldots, n$, there exist vectors $v_{j} \in$ $\mathbb{R}^{5}$, such that

$$
\sum_{j=1}^{n}\left\langle f_{\theta_{j}}, v_{j}\right\rangle=0 \quad \text { on } U_{x}
$$

Then the following hold:

$$
\begin{gathered}
\sum_{j=1}^{n} \exp \left(i \theta_{j}\right)\left(\kappa_{1}\left\langle T_{\theta} e_{3}, v_{j}\right\rangle-i \mu_{1}\left\langle T_{\theta} e_{4}, v_{j}\right\rangle\right)=0, \\
\kappa_{1} \sum_{j=1}^{n} \exp \left(2 i \theta_{j}\right)\left\langle f_{\theta_{j}}, v_{j}\right\rangle=L \sum_{j=1}^{n} \exp \left(i \theta_{j}\right)\left\langle T_{\theta} e_{4}, v_{j}\right\rangle
\end{gathered}
$$

away from the zeros of $\omega_{34}$, where $L=-E\left(\omega_{34}(E)\right)-3 i \omega_{12}(E) \omega_{34}(E)$, and

$$
\bar{E}\left(\sum_{j=1}^{n} \exp \left(i \theta_{j}\right)\left\langle T_{\theta} e_{4}, v_{j}\right\rangle\right)=-\omega_{34}(\bar{E}) \sum_{j=1}^{n} \exp \left(i \theta_{j}\right)\left\langle T_{\theta} e_{3}, v_{j}\right\rangle .
$$

Proof. Our assumption implies that

$$
\sum_{j=1}^{n}\left\langle d f_{\theta_{j}}, v_{j}\right\rangle=0
$$

Differentiating, using the Gauss formula, (4) and (7), we see that

$$
\sum_{j=1}^{n} \exp \left(i \theta_{j}\right)\left(\bar{H}_{3}\left\langle T_{\theta} e_{3}, v_{j}\right\rangle+\bar{H}_{4}\left\langle T_{\theta} e_{4}, v_{j}\right\rangle\right)=0 .
$$

Since $H_{3}=\kappa_{1}, H_{4}=i \mu_{1}$, the above immediately implies (8). 
Equations (3) yield

$$
\begin{aligned}
& E\left(\kappa_{1}\right)=-2 i \kappa_{1} \omega_{12}(E)+i \mu_{1} \omega_{34}(E), \\
& E\left(\mu_{1}\right)=-2 i \mu_{1} \omega_{12}(E)+i \kappa_{1} \omega_{34}(E) .
\end{aligned}
$$

Differentiating (8) with respect to $E$, using (5), (6), (12) and (13), we obtain

$$
\begin{aligned}
& \sum_{j=1}^{n} \exp \left(i \theta_{j}\right)\left(r\left\langle T_{\theta} e_{3}, v_{j}\right\rangle-s\left\langle T_{\theta} e_{4}, v_{j}\right\rangle\right) \\
& \quad=\frac{1}{2}\left(\kappa_{1}^{2}-\mu_{1}^{2}\right) \sum_{j=1}^{n} \exp \left(2 i \theta_{j}\right)\left\langle d f_{\theta_{j}}(\bar{E}), v_{j}\right\rangle,
\end{aligned}
$$

where $r=-i \kappa_{1} \omega_{12}(E)+i \mu_{1} \omega_{34}(E)$ and $s=\mu_{1} \omega_{12}(E)-\kappa_{1} \omega_{34}(E)$. Using (8), we have

$$
\sum_{j=1}^{n} \exp \left(i \theta_{j}\right)\left\langle T_{\theta} e_{4}, v_{j}\right\rangle=\frac{\kappa_{1}}{2 \omega_{34}(E)} \sum_{j=1}^{n} \exp \left(2 i \theta_{j}\right)\left\langle d f_{\theta_{j}}(\bar{E}), v_{j}\right\rangle .
$$

Differentiating (14) with respect to $E$, using (6), (8), the Gauss formula and (14), we find

$$
\begin{aligned}
& \left(E\left(\frac{\kappa_{1}}{\omega_{34}(E)}\right)-\frac{i \kappa_{1} \omega_{12}(E)}{\omega_{34}(E)}-i \mu_{1}\right) \sum_{j=1}^{n} \exp \left(2 i \theta_{j}\right)\left\langle d f_{\theta_{j}}(\bar{E}), v_{j}\right\rangle \\
& =\frac{2 \kappa_{1}}{\omega_{34}(E)} \sum_{j=1}^{n} \exp \left(2 i \theta_{j}\right)\left\langle f_{\theta_{j}}, v_{j}\right\rangle .
\end{aligned}
$$

By virtue of (12) and since $\kappa_{1}^{2}>\mu_{1}^{2}$ on $U_{x}$, the above is written as

$$
2 \omega_{34}(E) \sum_{j=1}^{n} \exp \left(2 i \theta_{j}\right)\left\langle f_{\theta_{j}}, v_{j}\right\rangle=L \sum_{j=1}^{n} \exp \left(2 i \theta_{j}\right)\left\langle d f_{\theta_{j}}(\bar{E}), v_{j}\right\rangle,
$$

where $L=-E\left(\omega_{34}(E)\right)-3 i \omega_{12}(E) \omega_{34}(E)$. Now (9) follows from (14) and (15). Moreover, we obtain using (6) that

$$
\begin{aligned}
\bar{E}\left(\sum_{j=1}^{n} \exp \left(i \theta_{j}\right)\left\langle T_{\theta} e_{4}, v_{j}\right\rangle\right)= & -i \mu_{1} \sum_{j=1}^{n}\left\langle d f_{\theta_{j}}(E), v_{j}\right\rangle \\
& -\omega_{34}(\bar{E}) \sum_{j=1}^{n} \exp \left(i \theta_{j}\right)\left\langle T_{\theta} e_{3}, v_{j}\right\rangle,
\end{aligned}
$$

which in view of (11) immediately yields (10).

We need the topological restrictions for minimal surfaces in $\mathbb{S}^{4}$ obtained by Eschenburg and Tribuzy [9]. To state their result, we recall that a nonzero function $a: M \rightarrow[0,+\infty)$ is called of absolute value type [7], [9] if locally $a=a_{0}|h|$, where $a_{0}$ is smooth and positive and $h$ is holomorphic. The zero 
set of such a function is isolated, and outside its zeros the function is smooth. The order $k \geq 1$ of any $x \in M$ with $a(x)=0$ is the order of $h$ at $x$. Let $N(a)$ be the sum of all orders for all zeros of $a$. Then $\Delta \log a$ is bounded on $M \backslash\{a=0\}$ and its integral satisfies

$$
\int_{M} \Delta \log a d A=-2 \pi N(a) .
$$

Lemma $8([9])$. Let $f: M \rightarrow \mathbb{S}^{4}$ be a compact minimal nonsuperminimal surface with Gaussian curvature $K$ and normal curvature $K_{N}$. Then the functions $a_{ \pm}=\left(1-K \pm K_{N}\right)^{1 / 2}$ are of absolute value type and the Euler numbers $\chi(M), \chi(N f)$ of the tangent and the normal bundle satisfy

$$
2 \chi(M) \pm \chi(N f)=-N\left(a_{\mp}\right) .
$$

Proof of Theorem 2. We assume that $f$ is not superminimal. According to Theorem 1, either $\mathcal{S}(f)$ is finite or $\mathcal{S}(f)=[0,2 \pi]$. Suppose to the contrary that $\mathcal{S}(f)=[0,2 \pi]$. We claim that the coordinate functions of the minimal surfaces $f_{\theta}, \theta \in[0, \pi)$, are linearly independent. Since these functions are eigenfunctions of the Laplace operator of $M$ with corresponding eigenvalue 2 , this contradicts the fact the eigenspaces of the Laplace operator are finite dimensional. To show that the coordinate functions are linearly independent, it is enough to prove that if

$$
\sum_{j=1}^{n}\left\langle f_{\theta_{j}}, v_{j}\right\rangle=0,
$$

for $0<\theta_{1}<\cdots<\theta_{n}<\pi$, then $v_{j}=0$ for all $1 \leq j \leq n$.

Assume to the contrary that $v_{j} \neq 0$ for all $1 \leq j \leq n$. Let $M_{1}=\left\{x_{1}, \ldots, x_{k}\right\}$ be the zero set of $\Phi$. Around each point $x \in M \backslash M_{1}$, we choose local complex coordinate $\left(U_{x}, z\right)$ and an orthonormal frame $\left\{e_{1}, e_{2}, e_{3}, e_{4}\right\}$ on $U_{x} \subset M \backslash M_{1}$ as in Lemma 6 . We consider the complex valued function

$$
\psi:=\left(\sum_{j=1}^{n} \exp \left(i \theta_{j}\right)\left\langle T_{\theta_{j}} e_{4}, v_{j}\right\rangle\right)^{2},
$$

where $T_{\theta_{j}}: N f \rightarrow N f_{\theta_{j}}$ is the bundle isomorphism of Lemma 5. Obviously $\psi$ is well-defined on $M \backslash M_{1}$. The second equation of (3) yields

$$
\omega_{34}(\bar{E})=\frac{i}{\kappa_{1}^{2}-\mu_{1}^{2}}\left(\kappa_{1} \bar{E}\left(\mu_{1}\right)-\mu_{1} \bar{E}\left(\kappa_{1}\right)\right) .
$$

Then (8) and (10) imply that $\bar{E}\left(\psi\left(1-\mu^{2} / \kappa^{2}\right)\right)=0$. Hence, the function $\psi\left(1-\mu^{2} / \kappa^{2}\right): M \backslash M_{1} \rightarrow \mathbb{C}$ is holomorphic. Since $\Psi$ is bounded, its isolated singularities are removable and consequently there exists a constant $c$ such that

$$
\psi\left(\kappa^{2}-\mu^{2}\right)=c \kappa^{2} \quad \text { on } M \backslash M_{1} .
$$


We claim that $c=0$. Indeed, if $\kappa\left(x_{l}\right)=\mu\left(x_{l}\right)>0$, for some $l$, then taking the limit in (17) along a sequence of points in $M \backslash M_{1}$ which converges to $x_{l}$, we deduce that $c=0$.

Suppose now that $\kappa\left(x_{l}\right)=\mu\left(x_{l}\right)=0$ for all $1 \leq l \leq k$. Let $(V, z)$ be a local complex coordinate around $x_{l}$ with $z\left(x_{l}\right)=0$. It is a well-known consequence of the Codazzi equation that

$$
B^{(2,0)}=B\left(\frac{\partial}{\partial z}, \frac{\partial}{\partial z}\right) d z^{2}
$$

is holomorphic as a $N f \otimes \mathbb{C}$-valued tensor field (cf. [10]). Since $B^{(2,0)}$ is not identically zero and $\left.B\right|_{x_{l}}=0$, we may write

$$
B^{(2,0)}=z^{m_{l}} \tilde{B}^{(2,0)} \quad \text { on } V
$$

where $m_{l}$ is a positive integer and $\tilde{B}^{(2,0)}$ is a tensor field of type $(2,0)$ with $\left.\tilde{B}^{(2,0)}\right|_{x_{l}} \neq 0$. We define the $N f$-valued tensor field $\tilde{B}:=\tilde{B}^{(2,0)}+\overline{\tilde{B}^{(2,0)}}$. Since its $(1,1)$-part vanishes, it follows easily that $\tilde{B}$ maps the unit circle on each tangent plane into an ellipse, on the corresponding normal space, whose length of the semi-axes are denoted by $\tilde{\kappa} \geq \tilde{\mu} \geq 0$. We also consider the differential form of type $(4,0)$

$$
\tilde{\Phi}:=\left\langle\tilde{B}^{(2,0)}, \tilde{B}^{(2,0)}\right\rangle
$$

which, in view of (18), is related to the Hopf differential of $f$ by $\Phi=z^{2 m_{l}} \tilde{\Phi}$. We split $\Phi$ and $\tilde{\Phi}$, with respect to arbitrary orthonormal frames $\left\{\xi_{1}, \xi_{2}\right\}$ and $\left\{\xi_{3}, \xi_{4}\right\}$ of $T M \mid V$ and $N f \mid V$, respectively as

$$
\begin{aligned}
\Phi & =\frac{1}{4}\left(\bar{H}_{3}^{2}+\bar{H}_{4}^{2}\right) \varphi^{4}=\frac{1}{4} k_{+} k_{-} \varphi^{4}, \\
\tilde{\Phi} & =\frac{1}{4}\left(\tilde{\tilde{H}}_{3}^{2}+\overline{\tilde{H}}_{4}^{2}\right) \varphi^{4}=\frac{1}{4} \tilde{k}_{+} \tilde{k}_{-} \varphi^{4},
\end{aligned}
$$

where

$$
\begin{aligned}
k_{ \pm} & =\bar{H}_{3} \pm i \bar{H}_{4}, \quad \tilde{k}_{ \pm}=\overline{\tilde{H}}_{3} \pm i \tilde{\tilde{H}}_{4}, \\
H_{\alpha} & =\left\langle B\left(\xi_{1}, \xi_{1}\right), \xi_{\alpha}\right\rangle+i\left\langle B\left(\xi_{1}, \xi_{2}\right), \xi_{\alpha}\right\rangle, \\
\tilde{H}_{\alpha} & =\left\langle\tilde{B}\left(\xi_{1}, \xi_{1}\right), \xi_{\alpha}\right\rangle+i\left\langle\tilde{B}\left(\xi_{1}, \xi_{2}\right), \xi_{\alpha}\right\rangle, \quad \alpha=3,4 .
\end{aligned}
$$

From (18), we obtain $\bar{H}_{\alpha}=z^{m_{l}} \tilde{H}_{\alpha}$, or equivalently, $k_{ \pm}=z^{m_{l}} \tilde{k}_{ \pm}$. Hence

$$
\kappa=|z|^{m_{l}} \tilde{\kappa}, \quad \mu=|z|^{m_{l}} \tilde{\mu} .
$$

Now (17) yields

$$
\psi\left(\tilde{\kappa}^{2}-\tilde{\mu}^{2}\right)=c \tilde{\kappa}^{2} \quad \text { on } V \backslash\left\{x_{l}\right\} .
$$

If $\tilde{\kappa}\left(x_{l}\right)>\tilde{\mu}\left(x_{l}\right)$ for all $1 \leq l \leq k$, then (19) implies that $N\left(a_{+}\right)=\sum_{l=1}^{k} m_{l}=$ $N\left(a_{-}\right)$. Hence, Lemma 8 yields $\chi(N f)=0$, which contradicts our assumption. Thus, $\tilde{\kappa}\left(x_{l}\right)=\tilde{\mu}\left(x_{l}\right)$ for some $1 \leq l \leq k$. Taking the limit in (20), along a 
sequence of points in $V \backslash\left\{x_{l}\right\}$ which converges to $x_{l}$, we obtain $c \tilde{\kappa}^{2}\left(x_{l}\right)=0$. Since $\left.\tilde{B}\right|_{x_{l}} \neq 0$, we infer that $c=0$.

From (17), we obtain $\psi=0$ on $M \backslash M_{1}$. Hence, (9) implies

$$
\sum_{j=1}^{n} \exp \left(2 i \theta_{j}\right)\left\langle f_{\theta_{j}}, v_{j}\right\rangle=0 .
$$

Combining this with (16), we have

$$
\sum_{j=2}^{n}\left\langle f_{\theta_{j}}, w_{j}\right\rangle=0,
$$

where $w_{j}:=\lambda_{j} v_{j} \neq 0, j=2, \ldots, n$, and $\lambda_{j}=\cos 2 \theta_{n}-\cos 2 \theta_{1}$ or $\lambda_{j}=\sin 2 \theta_{n}-$ $\sin 2 \theta_{1}$. By induction, we finally conclude that $\left\langle f_{\theta_{n}}, w\right\rangle=0$, for some nonzero vector $w$. So $f_{\theta_{n}}$ lies in a totally geodesic $\mathbb{S}^{3}$, contradiction. This concludes the proof of the theorem.

Proof of Corollary 3. Let $f_{\theta_{j}}, j=1, \ldots, n, 0=\theta_{0}<\theta_{1}<\cdots<\theta_{n} \leq 2 \pi$ is the maximal family of noncongruent minimal surfaces in $\mathbb{S}^{4}$ which are isometric to $f$ and have the same normal curvature. Since the second fundamental form of the minimal surfaces $f_{t}:=f \circ \varphi_{t}$ depends continuously on the parameter, we deduce that $f_{t}$ is congruent to exactly one of $f_{\theta_{j}}$ for all $t$. Since $f \circ \varphi_{0}=f$, we conclude that $f_{t}$ is congruent to $f$ for all $t$.

\section{REFERENCES}

[1] A. C. Asperti, Immersions of surfaces into 4-dimensional spaces with nonzero normal curvature, Ann. Mat. Pura Appl. (4) 125 (1980), 313-328. MR 0605213

[2] J. L. Barbosa, On minimal immersions of $\mathbb{S}^{2}$ into $\mathbb{S}^{2 m}$, Trans. Amer. Math. Soc. 210 (1975), 75-106. MR 0375166

[3] S. S. Chern, On the minimal immersions of the two-sphere in a space of constant curvature, Problems in analysis, Princeton University Press, Princeton, 1970, pp. 2740. MR 0362151

[4] H. Choi, W. Meeks and B. White, A rigidity theorem for properly embedded minimal surfaces in $\mathbb{R}^{3}$, J. Differential Geom. 32 (1990), 65-76. MR 1064865

[5] M. Dajczer and D. Gromoll, Gauss parametrizations and rigidity aspects of submanifolds, J. Differential Geom. 22 (1985), 1-12. MR 0826420

[6] M. Dajczer and D. Gromoll, Real Kähler submanifolds and uniqueness of the Gauss map, J. Differential Geom. 22 (1985), 13-28. MR 0826421

[7] J. H. Eschenburg, I. V. Guadalupe and R. Tribuzy, The fundamental equations of minimal surfaces in $\mathbb{C} P^{2}$, Math. Ann. 270 (1985), 571-598. MR 0776173

[8] J. H. Eschenburg and P. Quast, The spectral parameter of pluriharmonic maps, Bull. Lond. Math. Soc. 42 (2010), 229-236. MR 2601549

[9] J. H. Eschenburg and R. Tribuzy, Constant mean curvature surfaces in 4-space forms, Rend. Semin. Mat. Univ. Padova 79 (1988), 185-202. MR 0964030

[10] I. V. Guadalupe and L. Rodriguez, Normal curvature of surfaces in space forms, Pacific J. Math. 106 (1983), 95-103. MR 0694674

[11] R. Lashof and S. Smale, On the immersion of manifolds in euclidean space, Ann. of Math. (2) 68 (1958), 562-583. MR 0103478 
[12] J. Ramanathan, Rigidity of minimal surfaces in $\mathbb{S}^{3}$, Manuscripta Math. 60 (1988), 417-422. MR 0933472

[13] B. Smyth and G. Tinaglia, The number of constant mean curvature isometric immersions of a surface, Comment. Math. Helv. 88 (2013), 163-183. MR 3008916

[14] R. Tribuzy and I. V. Guadalupe, Minimal immersions of surfaces into 4-dimensional space forms, Rend. Semin. Mat. Univ. Padova 73 (1985), 1-13. MR 0799891

[15] T. Vlachos, Congruence of minimal surfaces and higher fundamental forms, Manuscripta Math. 110 (2003), 77-91. MR 1951801

Theodoros Vlachos, Department of Mathematics, University of Ioannina, 45110 Ioannina, Greece

E-mail address: tvlachos@uoi.gr 